\title{
Correlation between cognitive impairment during the acute phase of first cerebral infarction and development of long-term pseudobulbar affect
}

This article was published in the following Dove Press journal:

Neuropsychiatric Disease and Treatment

\section{Yuan Wang ${ }^{1,2}$ \\ Yuliang Wang ${ }^{2}$ \\ Wenbin $\mathrm{Ma}^{2}$ \\ Shujun $\mathrm{Lu}^{2}$ \\ Jinbo Chen² \\ Lili Cao'}

'Department of Neurology, Qilu Hospital of Shandong University, Jinan, People's Republic of China;

2Department of Neurology, Binzhou Medical University Hospital, Binzhou, People's Republic of China
Correspondence: Lili Cao

Department of Neurology, Qilu Hospital of Shandong University, 107 Jinan Culture Road, Jinan, Shandong 256600, People's

Republic of China

Tel+86531821691।4

Email121892349@qq.com
Purpose: The relationship between cognitive impairment during the acute phase of first cerebral infarction and the development of long-term pseudobulbar affect (PBA) has not been elucidated. Therefore, in this study, we aimed to determine if cognitive impairment during the acute phase of cerebral infarction will increase the risk of long-term post-infarction PBA.

Patients and methods: This was a nested case-control study using a prospective approach. A consecutive multicenter matched 1:1 case-control study of cognitive impairment cases following acute cerebral infarction ( $\mathrm{N}=26)$ with 26 sex-, education years-, and age-matched controls. Univariate and multivariate conditional logistic regression analyses were performed to study the clinical features and changes in cognitive domain as well as the risk factors for PBA.

Results: Long-term PBA was independently predicted by low Montreal cognitive assessment (MoCA) scores at baseline. Multivariable regression models showed that post-infarction low MoCA scores remained independent predictors of long-term PBA (odds ratio $[\mathrm{OR}]=0.72$; $95 \%$ confidence interval $[\mathrm{CI}]=0.54-0.95 ; P=0.018)$. Among all cognitive disorders, digit span test (DST) scores $(\mathrm{OR}=0.39 ; 95 \% \mathrm{CI}=0.16-0.91, P=0.030)$, StroopC time $(\mathrm{OR}=1.15 ; 95 \%$ $\mathrm{CI}=1.01-1.31 ; P=0.037)$, and clock-drawing task $(\mathrm{CDT})$ scores $(\mathrm{OR}=0.62 ; 95 \% \mathrm{CI}=0.42-0.90$; $P=0.013$ ) were found to be the independent risk factors for PBA.

Conclusion: Cognitive impairment during the acute phase of cerebral infarction increased the risk of cerebral infarction-induced long-term PBA. Development of PBA was closely associated with executive function, attention, and visuospatial disorder.

Keywords: cognitive impairment, pseudobulbar affect, cerebral infarction, neuropsychological tests, cognitive domain

\section{Introduction}

Pseudobulbar affect (PBA) is a disorder of emotional expression characterized by uncontrolled outburst of laughter or crying that lacks an appropriate environmental trigger and may be exaggerated or incongruent with the underlying emotional state. It is different from mood disorders, such as depression and bipolar disorders. ${ }^{1}$ It may result from a variety of neurological diseases, such as stroke, amyotrophic lateral sclerosis (ALS), traumatic brain injury, and multiple sclerosis. It most commonly happens after stroke, with an incidence of $11 \%-52 \%, 2,3$ it is most commonly noted during the 4th-6th week after the stroke, ${ }^{4}$ however, at various times of onset. After stroke, the incidence of PBA has been shown to be approximately $15 \%$ in the first month, $25 \%$ in the second month, and $11 \%$ in the 12 th month. Post-stroke PBA is more common in females than in males, with cerebral infarction occurring more frequently than cerebral hemorrhage. Pathological crying is most common after stroke, followed by pathological 
laughing and crying (PLC). ${ }^{5,6} \mathrm{PBA}$ is a common complication of neurological impairment, affecting survival satisfaction, social interaction, and rehabilitation processes in the patients with stroke. ${ }^{3,7}$ Hence, knowledge regarding the predictors of PBA after cerebral infarction is particularly important for the early diagnosis of the disease.

The acute manifestation of a stroke is not only hemiplegia but also cognitive impairment in the patients. According to the minimum-mental state examination (MMSE), the incidence of cognitive dysfunction 3 months after stroke ranges from $24 \%$ to $39 \%$. However, the incidence of cognitive dysfunction after stroke has been assessed as high as $96 \%$ using comprehensive neuropsychological tests. ${ }^{8}$ A latest Chinese study has shown that the incidence of post-stroke cognitive impairment (PSCI) is around $81 \%$ as measured by the Montreal cognitive assessment (MoCA). ${ }^{9}$ An observational study of acute stroke cases in People's Republic of China revealed that cognitive impairment is significantly and positively correlated with hemiplegia, ${ }^{10}$ which is known to have a significant effect on the prognosis of patients with stroke. While most of the studies have focused on the finding the association of cognitive impairment with hemiplegia, studies focusing on correlation between cognitive impairment and PBA are scarce. ${ }^{11}$ At present, it is still unclear whether cognitive impairment early after stroke is a risk factor for long-term PBA, independent of other well-known medical and demographic predictors.

Therefore, in this study, we aimed to test whether acute cognitive impairment is an independent risk factor for PBA 6 months after stroke. Furthermore, we examined which specific cognitive deficits in the early phase of stroke were associated with PBA.

\section{Patients and methods}

\section{Source of patients}

A total of 202 patients with first acute cerebral infarction treated at two medical centers in Binzhou district, Shandong Province, People's Republic of China, from September 2015 to January 2016 were recruited in this study. These patients had new clinical symptoms and signs, and infarction was confirmed by brain magnetic resonance imaging (MRI) and diffusion-weighted imaging (DWI). We further screened the patients according to the following criteria: 1) first acute infarction occurring within 7 days before admission; 2) age in between 18 and 85 years; 3 ) responsible foci on imaging were consistent with the clinical symptoms and signs; 4 ) confirmed presence of normal cognitive function before the disease onset; 5) ability to give consent; and 6) capable of cooperating with the examinations and participate in the neuropsychological tests.

Patients were excluded from this study if 1) they had severe consciousness disorders, such as coma and delirium; 2 ) they had a history of cognitive impairment ( $\geq 3.6$ points in the short form of informant questionnaire on cognitive decline in the elderly); 3) they had any reason (eg, language barrier or movement disorders) that prevented them from completing the neuropsychological examinations; 4) they had suffered from a severe disease of any of the vital organs, such as heart, liver, or kidneys; 5) they had suffered from other neurological diseases (eg, genetic degeneration, brain tumors, encephalitis, demyelination, Parkinson's disease, head trauma, or epilepsy); 6) they suffer from anxiety ( $>7$ Hamilton Anxiety Rating Scale), depression ( $>8$ Hamilton Anxiety Rating Scale), or other psychiatric disorders; or 7) they had a history of drug abuse or medications likely to influence the results (such as hormone replacement therapy). According to 1:1 matched case-control study design, the minimum size for the test was computed with the formula.

One hundred and fifty-seven patients were included in this study. Their baseline clinical data were collected, and they completed MoCA test.

\section{Ethical statement}

This study was approved by the Ethics Committee of Binzhou Medical University Hospital and all procedures performed in studies involving human participants were in accordance with the ethical standards of the ethics committee. All patients provided signed informed content before participating in this study.

\section{Study population}

Patients with infarction were divided into two groups according to their MoCA scores: cognitive impairment and noncognitive impairment. Given the significant relationship between MoCA score and educational background reported in previous studies, ${ }^{12,13}$ the optimal cutoff points were considered as 14 for illiterate individuals, 20 for individuals with $1-6$ years of education, and 25 for individuals with 7 or more years of education. ${ }^{14}$ Post-infarction cognitive impairment was considered to be present if the MoCA scores were below the aforementioned critical values. Finally, 109 patients were included in their representative cohorts.

This study was designed as a nested case-control study from a prospective cohort study. All study participants were invited for the follow-up assessment; the median follow-up 
period was 6 months. We conducted follow-up visits via phone or by outpatient visit using questionnaires to collect the data. After excluding patients with recurrent stroke, unavailable contact information, refusal to participate, and with severe complications, a total of 96 participants $(88.0 \%)$ (or their guardians) were identified and subsequently agreed to be interviewed as part of the follow-up study. The acute onset of infarction in patients was used as the start date for each patient, and the development of the PBA was considered as the endpoint. The primary outcome variable was the presence or absence of PBA. A neurologist administered a brief questionnaire to both patients and their relatives to establish the diagnosis of PBA according to Kim's criteria. ${ }^{15}$ PBA was diagnosed if the patients exhibited excessive or inappropriate laughing, crying, or both compared with their premorbid state. If both the patient and relative(s) who lived with the patient agreed that excessive or inappropriate laughing or crying occurred on two or more occasions beginning from the follow-up, the diagnosis of PBA was established. Follow-up assessments were performed by physicians who were blinded to the patient's hospital course. During a median follow-up period, we identified 26 incident cases of newly diagnosed PBA. Then, we randomly selected controls among the patients with post-infarction cognitive impairment who remained free from PBA and matched them to the case patients in a 1:1 ratio, according to age (within 2 year), sex, and education background. The final study cohort consisted of 52 patients (Figure 1).

\section{Neuropsychological assessment}

Other than the MoCA, neuropsychological tests were grouped into 4 cognitive domains: 1) attention - the mainland Chinese version of the digit span test (DST) of the Wechsler Adult Intelligence Scale (WAIS-Rc) was used; ${ }^{16}$ 2) memory - Auditory Verbal Learning Test-HuaShan version test (AVLT-H) was used to assess memory of the patients; ${ }^{17} 3$ ) executive functions - controllability and response inhibition of the patients was determined using part C of the short form of the Stroop test (24 items with 4 colors, StroopC test), ${ }^{18}$ and the mental flexibility of the patients was measured by the verbal fluency tests (VFTs) (using the animal fluency test) $;{ }^{19}$ and 4 ) the visuospatial construction ability of the patients was evaluated by the executive clock-drawing task (CDT) developed by Royall. ${ }^{20}$

\section{Radiological examination}

An MRI, including DWI and sequence of proton density, was performed on each patient with a 1.5-T system (Sonata; Siemens Medical, Erlangen, Germany) within 7 days of admission. A neurologist and a radiologist, who were blind to the psychiatric diagnoses, assessed the MRIs. The MRI assessment included the following: 1) brain infarcts: the presence of acute infarcts affecting the frontal, temporal,

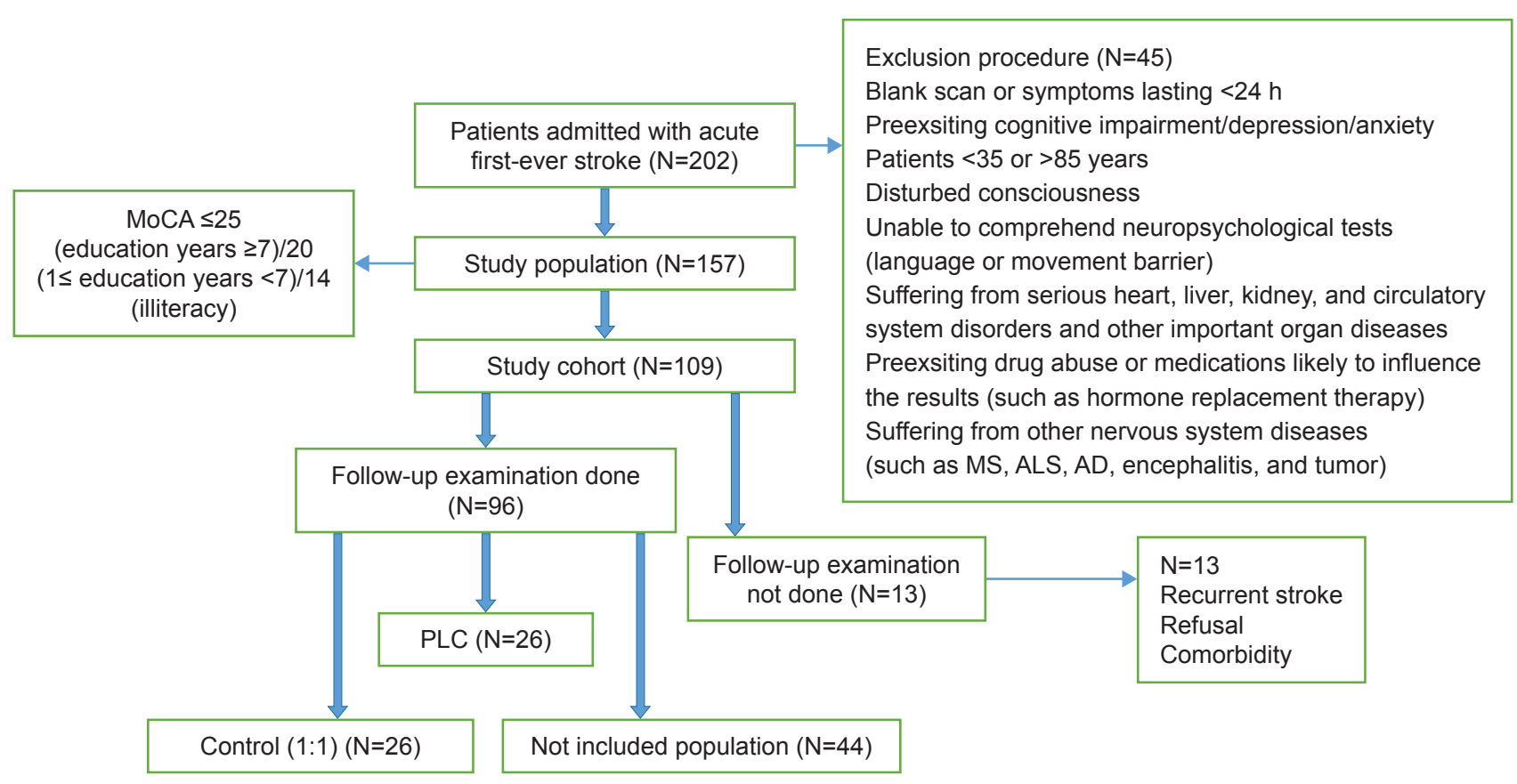

Figure I Flowchart of patient inclusion.

Abbreviations: PLC, pathological laughing and crying; MoCA, Montreal cognitive assessment; ALS, amyotrophic lateral sclerosis. 
parietal, and occipital lobes; basal ganglia; thalamus; brainstem; cerebellar and subcortical white matter region; 2) white matter lesions: the extent of white matter lesions was graded using Fazekas' 4-point scale. ${ }^{21}$ Periventricular lesions and deep white matter lesions were scored on proton density images.

\section{Statistical analysis}

Descriptive statistics are presented as frequencies, means, and standard deviation (SD). Differences between groups were examined with Fisher's exact test for proportions and with Student's $t$-test for continuous variables. In these analyses, the case and control subjects were matched by age, education years and sex within each center (1:1 pair matching). The associations between cognitive impairment in acute phase stroke and long-term PBA symptoms were investigated by conditional logistic regression analyses. In the first step, multivariate models were adjusted by white matter lesions, hypertension, diabetes, hyperlipidemia, smoking, drinking, and National Institute of Health Stroke Scale (NIHSS) total score. In the second step, we examined different cognitive domains at baseline in multivariable regression models. All analyses were performed with SPSS statistical software version 13.0 (SPSS Inc, Chicago, IL, USA) and SAS statistical software version 9.2 (SAS Institute Inc, Carey, NC, USA). A $P$-value $<0.05$ was accepted as indicating statistical significance.

\section{Results}

After the early phase of stroke, 26 (27.1\%) patients demonstrated PBA during follow-up. Table 1 shows univariate associations between potential predictor variables and long-term PBA. PBA was independently associated with low MoCA scores in the acute stage of stroke. There were no significant differences between the two groups with respect to age, sex, years of education, smoking, drinking, hypertension, diabetes, hyperlipidemia, NIHSS total scores, and the extent of white matter lesions. The PBA group had significantly more acute infarcts in the frontal lobe and basal ganglia. Table 2 summarizes the results of multiple stepwise logistic regression analysis. Multivariable regression models adjusting for age, sex, years of education, smoking, drinking, lesion location, NIHSS total score, the extent

Table I Predictors of long-term PBA: characteristics of the study subjects at baseline

\begin{tabular}{|c|c|c|c|c|c|c|c|c|c|c|c|}
\hline & \multicolumn{5}{|c|}{ Cases $(\mathbf{N}=\mathbf{2 6})$} & \multicolumn{5}{|c|}{ Controls $(\mathbf{N}=26)$} & \multirow[t]{2}{*}{$P$-value } \\
\hline & $\mathbf{N}$ & Mean & SD & $\mathbf{N}$ & $\%$ & $\mathbf{N}$ & Mean & SD & $\mathbf{N}$ & $\%$ & \\
\hline Age (years) & 26 & 62.27 & 7.54 & & & 26 & 61.46 & 7.46 & & & \\
\hline Gender (male/female) & 26 & & & $12 / 14$ & 46.15 & 26 & & & $12 / 14$ & 46.15 & \\
\hline Years of education & 26 & 7.30 & 2.80 & & & 26 & 7.40 & 2.80 & & & \\
\hline \multirow[t]{3}{*}{ White matter lesions } & 26 & & & & & 26 & & & & & \\
\hline & & 0.96 & 0.59 & & & & 0.88 & 0.70 & & & $0.658^{\mathrm{a}}$ \\
\hline & & 1.19 & 0.73 & & & & $\mathrm{I} .07$ & 0.73 & & & $0.556^{\mathrm{a}}$ \\
\hline Localization of acute infarct & 26 & & & & & 26 & & & & & \\
\hline Frontal lobe & & & & 10 & 38.46 & & & & 4 & 15.38 & $0.1164^{b}$ \\
\hline Basal ganglia region & & & & 9 & 34.62 & & & & 5 & 19.23 & $0.3487^{b}$ \\
\hline Frontal lobe and basal ganglia region & & & & 7 & 26.92 & & & & I & 3.85 & $0.0496^{b}$ \\
\hline Temporal lobe & & & & 4 & 15.38 & & & & 6 & 23.08 & $0.3497^{b}$ \\
\hline Parietal lobe & & & & 3 & 11.54 & & & & 4 & 15.38 & $1.000^{\mathrm{b}}$ \\
\hline Occipital lobe & & & & I & 3.85 & & & & 4 & 15.38 & $1.000^{\mathrm{b}}$ \\
\hline Brainstem & & & & 5 & 19.23 & & & & 2 & 7.69 & $0.4189^{b}$ \\
\hline Cerebellar & & & & 2 & 7.69 & & & & 2 & 7.69 & $1.000^{\mathrm{b}}$ \\
\hline Thalamus & & & & 3 & 11.54 & & & & 3 & 11.54 & $1.000^{\mathrm{b}}$ \\
\hline Subcortical white matter & & & & 7 & 26.92 & & & & 6 & 23.08 & $1.000^{\mathrm{b}}$ \\
\hline More than one location & & & & $\mathrm{II}$ & 42.31 & & & & 6 & 23.08 & $0.2367^{b}$ \\
\hline Smoking & 26 & & & 15 & 57.70 & 26 & & & 12 & 46.15 & $0.5793^{b}$ \\
\hline Drinking & 26 & & & 3 & 11.54 & 26 & & & 4 & 15.38 & $1.000^{\mathrm{b}}$ \\
\hline Hypertension & 26 & & & 20 & 76.92 & 26 & & & 17 & 65.38 & $0.5414^{b}$ \\
\hline Diabetes & 26 & & & 12 & 46.15 & 26 & & & 10 & 38.46 & $0.7793^{b}$ \\
\hline Hyperlipidemia & 26 & & & 21 & 80.77 & 26 & & & 19 & 73.08 & $0.7432^{b}$ \\
\hline NIHSS & 26 & 5.19 & 2.84 & & & 26 & 4.73 & 2.59 & & & $0.545^{\mathrm{a}}$ \\
\hline MMSE & 26 & 19.81 & 3.20 & & & 26 & 21.88 & 2.34 & & & $0.010^{\mathrm{a}}$ \\
\hline MoCA & 26 & 16.60 & 4.80 & & & 26 & 19.70 & 3.30 & & & $0.00 I^{a}$ \\
\hline
\end{tabular}

Notes: 'Student's $t$-test for continuous variables. 'Fisher's exact test for proportions. Variables shown as mean \pm SD or frequencies.

Abbreviations: PBA, pseudobulbar affect; SD, standard deviation; NIHSS, National Institute of Health Stroke Scale; MMSE, minimum-mental state examination; MoCA, Montreal cognitive assessment. 
Table 2 Predictors of long-term PBA:conditional logistic regression analysis

\begin{tabular}{llll}
\hline Variable & \multicolumn{3}{l}{ PBA at follow-up } \\
\cline { 2 - 4 } & OR & $\mathbf{9 5 \%} \mathbf{~ C l}$ & P-value \\
\hline MoCA & 0.715 & $0.54 I-0.945$ & 0.018 \\
MMSE & 0.461 & $0.24 I-0.882$ & 0.019 \\
\hline
\end{tabular}

Notes:ValuesareORand $95 \% \mathrm{Cl}$.Adjustedforsmoking, drinking, diabetes, hypertension, hyperlipidemia, NIHSS scores, white matter lesion, and lesion location.

Abbreviations: PBA, pseudobulbar affect; OR, odds ratio; $\mathrm{Cl}$, confidence interval; MoCA, Montreal cognitive assessment; MMSE, minimum-mental state examination; NIHSS, National Institute of Health Stroke Scale.

of white matter lesion, and vascular risk factors showed that post-infarction low MoCA scores remained independent predictors of long-term $\mathrm{PBA}$ (odds ratio $[\mathrm{OR}]=0.72$; $95 \%$ confidence interval $[\mathrm{CI}]=0.54-0.95 ; P=0.018)$.

Next, we examined which specific cognitive disorders at baseline were associated with PBA after the follow-up period (Table 3). Univariate logistic regression analysis showed that the PBA group had a significantly lower DST score, required more time to complete the StroopC test, and made more omission and errors in the CDT. There was no significant difference between groups in terms of memory as measured by the AVLT-H tests. Table 4 presents the results from multivariable regression models. After adjustment for age, sex, years of education, smoking, drinking, lesion location, NIHSS total score, the extent of white matter lesion and vascular risk factors, DST scores, StroopC time, and CDT scores remained significantly associated with PBA, respectively $(\mathrm{OR}=0.39,95 \% \mathrm{CI}=0.16-0.91, P=0.030$; $\mathrm{OR}=1.15,95 \% \mathrm{CI}=1.01-1.31, P=0.037 ; \mathrm{OR}=0.62,95 \%$ $\mathrm{CI}=0.42-0.90, P=0.013)$.

\section{Discussion}

In this study, the incidence of cognitive impairment during the acute phase of first cerebral infarction was as high

Table 3 Predictors of long-term PBA: cognitive domain characteristics of the study subjects at baseline

\begin{tabular}{|c|c|c|c|c|c|}
\hline \multirow[t]{2}{*}{ Study variable } & \multicolumn{2}{|c|}{$\begin{array}{l}\text { Cases } \\
(\mathrm{N}=26)\end{array}$} & \multicolumn{2}{|c|}{$\begin{array}{l}\text { Controls } \\
(\mathrm{N}=26) \\
\end{array}$} & \multirow[t]{2}{*}{$P$-value } \\
\hline & Mean & SD & Mean & SD & \\
\hline Delayed recall (3 min) & 4.07 & $\mathrm{I} .87$ & 4.46 & 1.42 & 0.4011 \\
\hline Delayed recall (30 min) & 3.42 & 1.92 & 4.19 & 1.39 & 0.1039 \\
\hline Recognition & 6.92 & 2.80 & 7.31 & 2.34 & 0.5889 \\
\hline StroopC-time & 50.38 & 18.57 & 40.73 & 12.41 & 0.0322 \\
\hline StroopC-error & 3.50 & 3.30 & 2.08 & 2.26 & 0.0763 \\
\hline VFT & 10.54 & 4.17 & 12.35 & 3.76 & 0.1065 \\
\hline DST & 4.07 & 0.97 & 4.73 & 1.15 & 0.0329 \\
\hline CDT & 6.69 & 2.69 & 8.38 & 2.90 & 0.0341 \\
\hline
\end{tabular}

Abbreviations: PBA, pseudobulbar affect; SD, standard deviation; VFT, verbal fluency test; DST, digit span test; CDT, clock-drawing task.
Table 4 Multivariate adjusted ORs of PBA for cognitive domains

\begin{tabular}{llll}
\hline Variable & \multicolumn{3}{l}{ PBA at follow-up } \\
\cline { 2 - 4 } & OR & $\mathbf{9 5 \%} \mathbf{C l}$ & P-value \\
\hline Delayed recall $(3 \mathrm{~min})$ & 0.641 & $0.401-1.024$ & $0.063 \mathrm{I}$ \\
DST & 0.386 & $0.163-0.911$ & 0.030 \\
StroopC-time & 1.148 & $1.009-1.308$ & 0.037 \\
VFT & 0.827 & $0.676-1.013$ & 0.067 \\
CDT & 0.616 & $0.420-0.904$ & 0.013 \\
\hline
\end{tabular}

Notes: Values are OR and $95 \% \mathrm{Cl}$. Adjusted for smoking, drinking, diabetes, hypertension, hyperlipidemia, NIHSS, white matter lesion, and lesion location. Abbreviations: PBA, pseudobulbar affect; OR, odds ratio; $\mathrm{Cl}$, confidence interval; DST, digit span test; VFT, verbal fluency test; CDT, clock-drawing task.

as $69 \%$. Likewise, the incidence of long-term PBA in the group with post-infarction cognitive impairment was up to $27 \%$. Previous reports have shown that antidepressants used to treat PBA can improve cognitive function in patients after stroke, ${ }^{22,23}$ which may indicate their correlation between PBA and cognitive impairment from one aspect. However, most studies on cognitive impairment in patients after infarction have focused on the long-term follow-up outcomes but rarely studied the effect of cognitive impairment during the acute phase on long-term PBA.

This study showed that the decline in cognitive function during the acute phase after cerebral infarction was an independent predictor of the development of long-term PBA. In addition, development of long-term PBA was associated with the severity of cognitive impairment. A previous study in ALS has shown that patients with PBA are more likely to have cognitive impairment. ${ }^{24}$ Comparison of PBA cases after stroke with controls also shows that mild cognitive impairment is an independent risk factor for PBA. ${ }^{11}$ These results were consistent with our findings. A possible explanation for these findings is that ischemic necrosis of the affected tissue in patients with acute cerebral infarction has damages to the neurological circuits of anatomical structures associated with cognitive function, ${ }^{25}$ these loops, such as prefrontal lobebasal ganglia loop, cerebral cortical-subcortical, and limbic system-diencephalon, intersect with the neural network of cortical-pontine-cerebellum loop, which is responsible for PBA. ${ }^{26}$ Therefore, cognitive impairment is often accompanied by frontal lobe-related information output barrier, leading to disorders of gate-control for motor expression of emotion which can manifest as PBA. ${ }^{26}$ Because PBA occurs in a wide variety of neurological disorders, specificity in the location of brain lesions appear to be more important to the pathogenesis of PBA. In our study, PBA and control groups had significant differences in frontal and basal ganglia infarcts. 
Previous studies have shown that the site and severity of cerebral infarction, hypertension, diabetes, and lifestyles are risk factors associated with cognitive impairment in the patients with cerebral infarction. ${ }^{27-32}$ However, this study suggested that although the above risk factors increased the risk of cerebral infarction-induced cognitive impairment, they were not closely related to the development of long-term PBA, which are unlikely to be useful as predictors of PBA.

We conducted neuropsychological tests to demonstrate a general loss of cognitive function during the acute phase of cerebral infarction. In addition, we further analyzed which specific cognitive domain was independently associated with the development of long-term PBA. We found that patients with visuospatial deficit, executive dysfunction, and attention deficit during the acute phase of stroke had relatively high incidence of long-term PBA after cerebral infarction. The comprehensive neuropsychological evaluation battery we conducted contains DST and StroopC test for attention and executive function. The CDT reflected not only the visuospatial function but also the executive function of patients with regards to numerical order integration and visuospatial organization (ie, frontal lobe function). The prefrontal region is closely associated with attention and executive function, and it is also involved in the processing of visuospatial memory. ${ }^{33}$ Therefore, lower function in these three specific cognitive domains suggests that long-term PBA after acute infarction is associated with damage to the loop related to the frontal lobe, such as frontal-basal gangliapontine-cerebellar loop. ${ }^{26}$ Studies of brain lesion location have suggested a wide-dispersed neural network involving frontal lobe in the pathophysiology of PBA. A recent study has supported this opinion that the prefrontal cortex is closely associated with PBA. ${ }^{34}$

Aharon-Peretz et al showed that despite the absence of clinically significant new vascular events, cognitive impairment is more and more serious with time prolonging after acute phase of infarction. ${ }^{35}$ This is consistent with the findings of Metter et al, ${ }^{36}$ who reported varying degrees of deficits in executive functioning and visuospatial construction ability after acute cerebral infarction. These may be due to the following: 1) subcortical lesions of related anatomical structures, resulting in secondary chronic cortical hypoperfusion and metabolic abnormalities; $; 3,372$ ) exacerbated latent small vessel disease in patients, such as the emergence of leukodystrophy or clinically silent new cerebral infarction; and 3) particularly evident aging-induced hemodynamic disorder in the prefrontal cortex, which is closely related to cognitive impairment. ${ }^{38}$ The development of long-term PBA after acute phase of infarction and the development of long-term cognitive impairment may share the same potential mechanism.

In summary, this study showed that cognitive impairment during the acute phase after cerebral infarction was an independent risk factor for long-term PBA. Development of PBA was closely associated with executive function, attention, and visuospatial disorder. Frontal lobe involvement of the infarct appears to play an important role.

The primary limitation of this study is its relatively small sample size, which reduced the statistical power in determining the difference between groups in certain tests and the correlation between the severity of PBA and the specific cognitive disorders. It also resulted in a low power in detecting the potential differences between groups in terms of frontal lobe infarcts and basal ganglia infarcts. Furthermore, the assessment of post-infarction cognitive impairment was made only once. As patients who had had recurrent infarction before the 6-month follow-up were not included in the study, the sample was biased to an unknown degree.

\section{Conclusion}

Cognitive impairment in the first weeks after cerebral infarction is an important risk factor for long-term PBA symptoms. Future studies are warranted to examine the relation between symptoms of PBA and cognitive impairment in more detail.

\section{Acknowledgments}

The authors wish to thank Qilu Hospital of Shandong University, Binzhou Medical University Hospital, and the Shandong Provincial Natural Science Foundation of China, for financially supporting this research under contract number ZR2016HM68.

\section{Author contributions}

All authors contributed toward data analysis, drafting and critically revising the paper, gave final approval of the version to be published, and agree to be accountable for all aspects of the work.

\section{Disclosure}

The authors report no conflicts of interest in this work.

\section{References}

1. Parvizi J, Arciniegas DB, Bernardini GL, et al. Diagnosis and management of pathological laughter and crying. Mayo Clin Proc. 2006; 81(11):1482-1486.

2. Parvizi J, Coburn KL, Shillcutt SD, Coffey CE, Lauterbach EC, Mendez MF. Neuroanatomy of pathological laughing and crying: a report of the American Neuropsychiatric Association Committee on Research. J Neuropsychiatry Clin Neurosci. 2009;21(1):75-87.

3. Wortzel HS, Oster TJ, Anderson CA, Arciniegas DB. Pathological laughing and crying: epidemiology, pathophysiology and treatment. CNS Drugs. 2008;22(7):531-545. 
4. Mukand J, Kaplan M, Senno RG, Bishop DS. Pathological crying and laughing: treatment with sertraline. Arch Phys Med Rehabil. 1996; 77(12):1309-1311.

5. Kim JS, Choi-Kwon S. Poststroke depression and emotional incontinence: correlation with lesion location. Neurology. 2000;54(9):1805-1810.

6. Yun SP, Jung WS, Park SU, et al. Hwangryunhaedogtang (huanglianjiedutang) treatment for pathological laughter after stroke and importance of patterns identification: a preliminary study. Am J Chin Med. 2007; 35(5):725-733.

7. Kasprisin A. Alternative cognitive therapy for emotional instability (pathologic laughing and crying). Phys Med Rehabil Clin N Am. 2004; 15(4):883-917, vii-viii.

8. Sun JH, Tan L, Yu JT. Post-stroke cognitive impairment: epidemiology, mechanisms and management. Ann Transl Med. 2014;2(8):80.

9. Qu Y, Zhuo L, Li N, et al. Prevalence of post-stroke cognitive impairment in China: a community-based, cross-sectional study. PLoS One. 2015;10(4):e0122864.

10. Zhong XP, Geng JG, Lu Y, Wang Q, Tan L. Correlation of cognitive dysfunction with hemiplegia and aphasia in patients with acute stroke. Chin J Clin Rehabil. 2006;10(46):181-183.

11. Wang G, Teng F, Chen Y, et al. Clinical features and related factors of poststroke pathological laughing and crying: a case-control study. J Stroke Cerebrovasc Dis. 2016;25(3):556-564.

12. Luis CA, Keegan AP, Mullan M. Cross validation of the Montreal Cognitive Assessment in community dwelling older adults residing in the Southeastern US. Int J Geriatr Psychiatry. 2009;24(2):197-201.

13. Smith T, Gildeh N, Holmes C. The Montreal Cognitive Assessment: validity and utility in a memory clinic setting. Can J Psychiatry. 2007; 52(5):329-332.

14. Lu J, Li D, Li F, et al. Montreal cognitive assessment in detecting cognitive impairment in Chinese elderly individuals: a population-based study. J Geriatr Psychiatry Neurol. 2011;24(4):184-190.

15. Choi-Kwon S, Han SW, Kwon SU, Kang DW, Choi JM, Kim JS. Fluoxetine treatment in poststroke depression, emotional incontinence, and anger proneness: a double-blind, placebo-controlled study. Stroke. 2006;37(1):156-161.

16. Dai XY, Ryan JJ, Paolo AM, Harrington RG. Factor analysis of the mainland Chinese version of the Wechsler Adult Intelligence Scale (WAIS-RC) in a brain-damaged sample. Int J Neurosci. 1990;55(2-4) 107-111.

17. Qinjie L, Ya M, Yuan Z. Auditory verbal learning test-HuaShan version in the diagnosis of amnestic mild cognitive impairment. Geriatr Health Care. 2016;22:282-285.

18. Jokinen H, Kalska H, Mäntylä R, et al. White matter hyperintensities as a predictor of neuropsychological deficits post-stroke. J Neurol Neurosurg Psychiatry. 2005;76(9):1229-1233.

19. Gorissen JP. [The recall of information from memory by patients with memory disorders]. Tijdschr Gerontol Geriatr. 1987;18(6):327-333. Dutch [with English abstract].

20. Sunderland T, Hill JL, Mellow AM, et al. Clock drawing in Alzheimer's disease. A novel measure of dementia severity. J Am Geriatr Soc. 1989; 37(8):725-729.

21. Fazekas F, Chawluk JB, Alavi A, Hurtig HI, Zimmerman RA. MR signal abnormalities at $1.5 \mathrm{~T}$ in Alzheimer's dementia and normal aging. AJR Am J Roentgenol. 1987;149(2):351-356.
22. Kimura M, Robinson RG, Kosier JT. Treatment of cognitive impairment after poststroke depression: a double-blind treatment trial. Stroke. 2000;31(7):1482-1486.

23. Narushima K, Chan KL, Kosier JT, Robinson RG. Does cognitive recovery after treatment of poststroke depression last? A 2-year follow-up of cognitive function associated with poststroke depression. Am J Psychiatry. 2003;160(6):1157-1162.

24. McCullagh S, Moore M, Gawel M, Feinstein A. Pathological laughing and crying in amyotrophic lateral sclerosis: an association with prefrontal cognitive dysfunction. J Neurol Sci. 1999;169(1-2):43-48.

25. Hillis AE, Barker PB, Wityk RJ, et al. Variability in subcortical aphasia is due to variable sites of cortical hypoperfusion. Brain Lang. 2004; 89(3):524-530.

26. Miller A, Pratt H, Schiffer RB. Pseudobulbar affect: the spectrum of clinical presentations, etiologies and treatments. Expert Rev Neurother. 2011;11(7):1077-1088.

27. Al-Qazzaz NK, Ali SH, Ahmad SA, Islam S, Mohamad K. Cognitive impairment and memory dysfunction after a stroke diagnosis: a post-stroke memory assessment. Neuropsychiatr Dis Treat. 2014;10: 1677-1691.

28. Pathak A, Hanon O, Negre-Pages L, Sevenier F; OSCAR Investigators. Rationale, design and methods of the OSCAR study: observational study on cognitive function and systolic blood pressure reduction in hypertensive patients. Fundam Clin Pharmacol. 2007;21(2):199-205.

29. Nagai M, Hoshide S, Kario K. Hypertension and dementia. Am J Hypertens. 2010;23(2):116-124.

30. Dufouil C, Richard F, Fiévet N, et al. APOE genotype, cholesterol level, lipid-lowering treatment, and dementia: the Three-City Study. Neurology. 2005;64(9):1531-1538.

31. Kalaria RN, Ballard C. Stroke and cognition. Curr Atheroscler Rep 2001;3(4):334-339.

32. Reitz C, Luchsinger J, Tang MX, Mayeux R. Effect of smoking and time on cognitive function in the elderly without dementia. Neurology. 2005;65(6):870-875.

33. Sampath D, Sathyanesan M, Newton SS. Cognitive dysfunction in major depression and Alzheimer's disease is associated with hippocampalprefrontal cortex dysconnectivity. Neuropsychiatr Dis Treat. 2017;13: 1509-1519.

34. Picó-Pérez M, Radua J, Steward T, Menchón JM, Soriano-Mas C. Emotion regulation in mood and anxiety disorders: a meta-analysis of fMRI cognitive reappraisal studies. Prog Neuropsychopharmacol Biol Psychiatry. 2017;79(Pt B):96-104.

35. Aharon-Peretz J, Daskovski E, Mashiach T, Tomer R. Natural history of dementia associated with lacunar infarctions. J Neurol Sci. 2002 203-204:53-55.

36. Metter EJ, Hanson WR, Jackson CA, et al. Temporoparietal cortex in aphasia. Evidence from positron emission tomography. Arch Neurol. 1990;47(11):1235-1238.

37. Weiller C, Ringelstein EB, Reiche W, Thron A, Buell U. The large striatocapsular infarct. A clinical and pathophysiological entity. Arch Neurol. 1990;47(10):1085-1091.

38. Agbangla NF, Audiffren M, Albinet CT. Use of near-infrared spectroscopy in the investigation of brain activation during cognitive aging: a systematic review of an emerging area of research. Ageing Res Rev. 2017;38:52-66.
Neuropsychiatric Disease and Treatment

\section{Publish your work in this journal}

Neuropsychiatric Disease and Treatment is an international, peerreviewed journal of clinical therapeutics and pharmacology focusing on concise rapid reporting of clinical or pre-clinical studies on a range of neuropsychiatric and neurological disorders. This journa is indexed on PubMed Central, the 'PsycINFO' database and CAS,

\section{Dovepress}

and is the official journal of The International Neuropsychiatric Association (INA). The manuscript management system is completely online and includes a very quick and fair peer-review system, which is all easy to use. Visit http://www.dovepress.com/testimonials.php to read real quotes from published authors. 